\title{
Multipower Variation and Stochastic Volatility
}

\author{
Ole E. BARNDORFF-NIELSEN \\ Department of Mathematical Sciences, \\ University of Aarhus, Ny Munkegade, DK-8000 Aarhus C, Denmark \\ oebn@imf .au.dk \\ NEIL SHEPHARD \\ Nuffield College, Oxford OX1 1NF, U.K. \\ neil.shephard@nuf.ox.ac.uk
}

November 18, 2004

\begin{abstract}
In this brief note we review some of our recent results on the use of high frequency financial data to estimate objects like integrated variance in stochastic volatility models. Interesting issues include multipower variation, jumps and market microstructure effects.
\end{abstract}

\section{Contents}

1 Introduction 1

2 Multipower variation 2

3 MPV for $\mathcal{B S M} \quad 3$

4 MPV for $\mathcal{B S M}+$ jump process 4

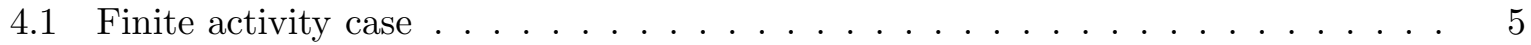

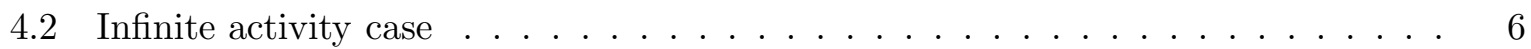

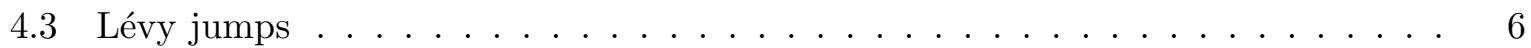

$\begin{array}{llr}5 & \text { Microstructure noise } & 7\end{array}$

6 Acknowledgments $\quad 8$

\section{Introduction}

This paper briefly summarises some recent and ongoing work concerning inference on stochastic volatility (see, for example, the reviews in Ghysels, Harvey, and Renault (1996) and Shephard (2005)), with the focus on multipower variation as a tool for such inference.

We assume that the $\log$ price process is of the form $X=Y+Z$ where $Y$ is an Brownian semimartingale $(\mathcal{B S M})$

$$
Y_{t}=Y_{0}+\int_{0}^{t} a_{u} \mathrm{~d} u+\int_{0}^{t} \sigma_{u} \mathrm{~d} W_{u}
$$

whose quadratic variation $[Y]$, which embodies the volatile character of $Y$, is the main object of interest, while $Z$ expresses effects that may be considered in some sense extraneous to the basic dynamics of the financial market. The process $Z$ may be a jump process, representing 
for instance the impacts of macroeconomic announcements, or it could represent microstructure noise.

In (1) $W$ is a Brownian motion, the volatility process $\sigma$ is assumed to be positive and càdlàg, $a$ is predictable and locally bounded, and we have the well known result that the quadratic variation of $Y$ satisfies

$$
[Y]_{t}=\int_{0}^{t} \sigma_{u}^{2} \mathrm{~d} u
$$

We shall write, for arbitrary $r>0$,

$$
\sigma_{t}^{r *}=\int_{0}^{t} \sigma_{u}^{r} \mathrm{~d} u
$$

and we wish to device inference procedures for these quantities, particularly for $\sigma_{t}^{2 *}\left(=[Y]_{t}\right)$.

Although the above formulation is in terms of univariate processes, much of the theory extends rather readily to a general multivariate setting. However, we shall not consider this further here but refer to the papers Barndorff-Nielsen and Shephard (2004a), Barndorff-Nielsen, Jacod, and Shephard (2004) and Barndorff-Nielsen, Graversen, Jacod, Podolskij, and Shephard (2004). We shall further restrict attention to equidistant sampling schemes; the situation under more general schemes are discussed in Barndorff-Nielsen and Shephard (2005) and Woerner (2004). See also Mykland and Zhang (2005).

After introducing the concepts of multipower variation (MPV) and generalised multipower variation in Section 2, we discuss, in Section 3, applications of MPV to inference on volatility under $\mathcal{B S M}$ models (that is, there we suppose that $Z=0$ ). Section 4 treats applications of MPV to cases where $Z$ is a jump process, both for finite and infinite activity scenarios. The final Section 5 indicates some work in progress concerning the impact of microstructure noise.

For numerical and empirical work and illustrations of the theoretical results presented here we additionally refer to Barndorff-Nielsen, Hansen, Lunde, and Shephard (2004), Barndorff-Nielsen, Hansen, Lunde, and Shephard (2005), Barndorff-Nielsen and Shephard (2002), Barndorff-Nielsen and Shephard (2003), Barndorff-Nielsen and Shephard (2004b) and Barndorff-Nielsen, Shephard, and Winkel (2004).

\section{Multipower variation}

For arbitrary continuous time processes $X=\left\{X_{t}\right\}_{t \geq 0}$ and equidistant subdivisions of time with lag $\delta>0$ we define the $\delta$-discretisation of $X$ by

$$
X_{\delta, t}=X_{t}-X_{\lfloor t / \delta\rfloor}
$$

where, as usual, $\lfloor s\rfloor$ indicates the largest integer less than or equal to a real number $s$. Furthermore, we introduce the realised multipower variation (MPV) of order $m$ for $X$ by

$$
\left[X_{\delta}\right]_{t}^{[\mathbf{r}]}=\left[X_{\delta}\right]_{t}^{\left[r_{1}, \ldots, r_{m}\right]}=\left[X_{\delta}, \ldots, X_{\delta}\right]_{t}^{\left[r_{1}, \ldots, r_{m}\right]}=\sum_{j=m}^{\lfloor t / \delta\rfloor}\left|x_{j-m+1}\right|^{r_{1}} \cdots\left|x_{j}\right|^{r_{m}}
$$

where $\mathbf{r}$ is short for $r_{1}, \ldots, r_{m}$, the $r_{l}$ being nonnegative, and

$$
x_{j}=X_{j \delta}-X_{(j-1) \delta} .
$$

We shall also use the normalised version of realised MPV, defined by

$$
\left\{X_{\delta}\right\}_{t}^{[\mathbf{r}]}=\left\{X_{\delta}\right\}_{t}^{\left[r_{1}, \ldots, r_{m}\right]}=\left\{X_{\delta}, \ldots, X_{\delta}\right\}_{t}^{\left[r_{1}, \ldots, r_{m}\right]}=\delta^{1-r_{+} / 2}\left[X_{\delta}\right]_{t}^{[\mathbf{r}]}
$$

where $r_{+}=r_{1}+\cdots+r_{m}$. 
In particular we will discuss applications of the power, bipower, and tripower variations (PV, BPV and TPV):

$$
\begin{gathered}
{\left[X_{\delta}\right]_{t}^{[r]}=\sum_{j=1}^{\lfloor t / \delta\rfloor}|x|^{r}} \\
{\left[X_{\delta}\right]_{t}^{[r, s]}=\sum_{j=2}^{\lfloor t / \delta\rfloor}\left|x_{j-1}\right|^{r}|x|^{s}} \\
{\left[X_{\delta}\right]_{t}^{[r, s, u]}=\sum_{j=3}^{\lfloor t / \delta\rfloor}\left|x_{j-2}\right|^{r}\left|x_{j-1}\right|^{s}\left|x_{j}\right|^{u} .}
\end{gathered}
$$

In the recent paper Barndorff-Nielsen, Graversen, Jacod, Podolskij, and Shephard (2004) the concept of MPV is generalised to generalised multipover variation where one considers realised objects of the form

$$
\sum_{j=m}^{\lfloor t / \delta\rfloor} g_{1}\left(\delta^{-1 / 2} x_{j-m+1}\right) \cdots g_{m}\left(\delta^{-1 / 2} x_{j}\right)
$$

where $g_{1}, \ldots, g_{m}$ are real functions satisfying certain regularity conditions, powers of absolute values being a special case. While this generalisation opens up further potential for applications, the associated central limit theory for (multivariate) $\mathcal{B S M}$ models, as established in BarndorffNielsen, Graversen, Jacod, Podolskij, and Shephard (2004), is in effect not more (or less) difficult than for the MPV case. In the following Section we draw on results from Barndorff-Nielsen, Graversen, Jacod, Podolskij, and Shephard (2004) to establish feasible limit theory for multipower variation under the $\mathcal{B S M}$ specification.

\section{3. $\mathrm{MPV}$ for $\mathcal{B S M}$}

Let $Y$ be a Brownian semimartingale as defined in Section 1. Important special cases are

$$
Y_{t}=Y_{0}+\int_{0}^{t} a\left(s, Y_{s}\right) \mathrm{d} s+\int_{0}^{t} \sigma\left(s, Y_{s}\right) \mathrm{d} W_{s},
$$

and

$$
Y_{t}=Y_{0}+\int_{0}^{t} a_{u} \mathrm{~d} u+\int_{0}^{t} \sigma_{u} \mathrm{~d} W_{u},
$$

with the volatility process $\sigma$ satisfying a stochastic differential equation of the form

$$
\sigma_{t}^{2}=\sigma_{0}^{2}+\int_{0}^{t} a_{s}^{\prime} \mathrm{d} s+\int_{0}^{t} \sigma_{s-}^{\prime} \mathrm{d} V_{s}+\int_{0}^{t} v_{s-} \mathrm{d} Z_{s}
$$

where $a^{\prime}, \sigma^{\prime}, v$ are adapted càdlàg processes, $V$ is a Brownian motion, possibly correlated with $W$, and $Z$ is a Lévy process. This second structure encompasses both the models of Heston type and those of non-Gaussian OU-based type introduced by Barndorff-Nielsen and Shephard (2001).

Without further assumptions we then have the following convergence in probability (CiP) and central limit theorem (CLT) for MPV.

Theorem As $\delta \rightarrow 0$

$$
\left\{Y_{\delta}, \ldots, Y_{\delta}\right\}_{t}^{\left[r_{1}, \ldots, r_{m}\right]} \stackrel{p}{\rightarrow} \mu_{r_{1}} \cdots \mu_{r_{m}} \sigma_{t}^{r_{+} *}
$$


and

$$
\delta^{-1 / 2}\left(\left\{Y_{\delta}, \ldots, Y_{\delta}\right\}_{t}^{\left[r_{1}, \ldots, r_{m}\right]}-\sigma_{t}^{r_{+} *}\right) \stackrel{l a w}{\longrightarrow} \sqrt{\nu_{\mathbf{r}}} \int_{0}^{t} \sigma_{u}^{r_{+}} \mathrm{d} B_{u}
$$

where $B$ is a Brownian motion which is independent of $Y$ and where

$$
\nu_{\mathbf{r}}=\prod_{l=1}^{m} \mu_{2 r_{l}}-(2 m-1) \prod_{l=1}^{m} \mu_{r_{l}}^{2}+2 \sum_{k=1}^{m-1} \prod_{l=1}^{k} \mu_{r_{l}} \prod_{l=m-k+1}^{m} \mu_{r_{l}} \prod_{l=1}^{m-k} \mu_{r_{l}+r_{l+k}}
$$

and $\mu_{r}=\mathrm{E}\left\{|u|^{r}\right\}$ for $u \sim N(0,1)$. The convergence in (3) is in fact stable as processes, which is stronger than convergence in law.

This theorem is a special case of the results established in Barndorff-Nielsen, Graversen, Jacod, Podolskij, and Shephard (2004). The proofs given there are (unavoidably) rather longwinded and use advanced stochastic analysis. An explanatory simpler version will be given in Barndorff-Nielsen, Jacod, and Shephard (2004).

The independence between $Y$ and $B$ is crucial for the possibility to establish statistically feasible CLT results, such as the following :

$$
\frac{\mu_{\mathbf{r}}^{-1}\left\{Y_{\delta}\right\}_{t}^{[\mathbf{r}]}-\sigma_{t}^{r_{+*}}}{\delta^{1 / 2} \mu_{\mathbf{r}}^{-1} \sqrt{\nu_{\mathbf{r}} \mu_{\mathbf{s}}^{-1}\left\{Y_{\delta}\right\}_{t}^{[\mathbf{s}]}}} \stackrel{\operatorname{law}}{\longrightarrow} N(0,1)
$$

where

$$
\mu_{\mathbf{r}}=\prod_{l=1}^{m} \mu_{r_{l}}
$$

and $\mathbf{s}=\left(s_{1}, \ldots, s_{m}\right)$ with $s_{+}=2 r_{+}$.

In particular, note that for realised PV, BPV and TPV in the case where $r_{+}=2, r_{j}=1 / r_{+}$, then for example,

$$
\begin{gathered}
\frac{\left\{Y_{\delta}\right\}_{t}^{[2]}-\sigma_{t}^{2 *}}{\delta^{1 / 2} \sqrt{\nu_{2} \mu_{4}^{-1}\left\{Y_{\delta}\right\}_{t}^{[4]}}} \stackrel{\text { law }}{\rightarrow} N(0,1), \\
\frac{\mu_{1}^{-2}\left[Y_{\delta}^{[1,1,1]}-\sigma_{t}^{2 *}\right.}{\delta^{1 / 2} \mu_{1}^{-4} \sqrt{\nu_{2,2}\left\{Y_{\delta}\right\}_{t}^{[1,1,1,1]}}} \stackrel{\text { law }}{\rightarrow} N(0,1)
\end{gathered}
$$

and

$$
\frac{\mu_{2 / 3}^{-3}\left[Y_{\delta}\right]_{t}^{[2 / 3,2 / 3,2 / 3]}-\sigma_{t}^{2 *}}{\delta^{1 / 2} \mu_{2 / 3}^{-6} \sqrt{\nu_{4 / 3,4 / 3,4 / 3}\left\{Y_{\delta}\right\}_{t}^{[2 / 3,2 / 3,2 / 3,2 / 3,2 / 3,2 / 3]}}} \stackrel{\text { law }}{\rightarrow} N(0,1) .
$$

\section{4. $\mathrm{MPV}$ for $\mathcal{B S M}+$ jump process}

We now consider various extensions of the above results to one-dimensional processes of the form

$$
X=Y+Z
$$

where $Y \in \mathcal{B S M}$ while $Z$ is a process exhibiting jumps. The processes $Y$ and $Z$ are not assumed to be independent. Our discussion is based on Barndorff-Nielsen, Shephard, and Winkel (2004) and is related to Barndorff-Nielsen and Shephard (2004b) and Woerner (2004).

We assume that $Y$ satisfies (2) or (3) for MPV and consider to which extent this limiting behaviour remains the same when $Z$ is added to $Y$, i.e. whether the influence of $Z$ is negligible (in this respect). 
In other words, we ask whether:

for the $\mathrm{CiP}$ case

$$
\left\{X_{\delta}, \ldots, X_{\delta}\right\}^{\left[r_{1}, \ldots, r_{m}\right]}-\left\{Y_{\delta}, \ldots, Y_{\delta}\right\}^{\left[r_{1}, \ldots, r_{m}\right]}=o_{p}(1)
$$

for the CLT case

$$
\left\{X_{\delta}, \ldots, X_{\delta}\right\}^{\left[r_{1}, \ldots, r_{m}\right]}-\left\{Y_{\delta}, \ldots, Y_{\delta}\right\}^{\left[r_{1}, \ldots, r_{m}\right]}=o_{p}\left(\delta^{1 / 2}\right) .
$$

We shall use the fact that $Y$ satisfies

$$
\delta^{-1 / 2}\left|Y_{j \delta}-Y_{(j-1) \delta}\right|=O_{p}\left(|\log \delta|^{1 / 2}\right)
$$

uniformly in $j$. We write $\max r$ for $\max \left\{r_{1}, \ldots, r_{m}\right\}$.

\subsection{Finite activity case}

When $Z$ is a finite activity jump process then pathwise the number of jumps of $Z$ is finite and, for sufficiently small $\delta$, none of the additive terms in $\left[X_{\delta}, \ldots, X_{\delta}\right]^{\left[r_{1}, \ldots, r_{m}\right]}$ involves more than one jump.

Each of the terms in $\left[X_{\delta}, \ldots, X_{\delta}\right]^{\left[r_{1}, \ldots, r_{m}\right]}$ that contains no jumps is of order

$$
O_{p}\left((|\log \delta|)^{r_{+} / 2}\right)
$$

and any of the terms that do include a jump is of order

$$
O_{p}\left((|\log \delta|)^{\left(r_{+}-\max r\right)}\right)
$$

Hence

$$
\begin{aligned}
\delta^{1-r_{+} / 2}\left(\left[X_{\delta}\right]^{[r]}-\left[Y_{\delta}\right]^{[r]}\right) & =\delta^{1-r_{+} / 2} O_{p}\left((\delta|\log \delta|)^{\left(r_{+}-\max r\right) / 2}\right) \\
& =O_{p}\left(\delta^{1-\max r / 2}|\log \delta|^{\left(r_{+}-\max r\right) / 2}\right)
\end{aligned}
$$

So:

- $\mathrm{CiP}$ is not influenced by $Z$ so long as $\max r<2$, while CLT continues to hold so long as $\max r<1$.

The bound $\max r<2$ seems quite a tight condition for when $m=1$ and $r=2$

$$
\left[X_{\delta}\right]^{[2]} \stackrel{p}{\rightarrow}[Y]+[Z]
$$

i.e. jumps do impact the limit.

The above CiP and CLT results mean that we can use multipower variation to make inference about $\sigma_{t}^{2 *}$, integrated variance, in the presence of finite activity jump processes so long as $\max r<1$ and $r_{+}=2$.

An example of this is where $m=3$ and we take $r_{1}=r_{2}=r_{3}=2 / 3$, that is using TPV Tripower Variation, cf. relation (5) above. 


\subsection{Infinite activity case}

In discussing $\mathrm{CiP}$ and CLT for the case where $Z$ exhibits infinite activity, i.e. infinitely many jumps in any finite time interval, we shall for simplicity restrict consideration to the case $r_{1}=$ $\cdots=r_{m}=r$. Detailed calculations, using classical inequalities, show that

- for MPVCiP it suffices that

$$
\begin{gathered}
\delta^{1-m r / 2}\left[Z_{\delta}, \ldots, Z_{\delta}\right]^{[r, \ldots, r]}=o_{p}(1) \\
\delta^{1-(m-1) r / 2}|\log \delta|\left[Z_{\delta}, \ldots, Z_{\delta}\right]^{[r, \ldots, r]}\left[\left(\begin{array}{c}
m \\
1
\end{array}\right)\right]=o_{p}(1) \\
\ldots \ldots . \\
\delta^{1-r / 2}|\log \delta|^{m-1}\left[Z_{\delta}\right]^{[r]}\left[\left(\begin{array}{c}
m \\
1
\end{array}\right)\right]=o_{p}(1) .
\end{gathered}
$$

- For MPVCLT it suffices that $r \leq 1$ and

$$
\begin{gathered}
\delta^{(1-m r) / 2}\left[Z_{\delta}, \ldots, Z_{\delta}\right]^{[r, \ldots, r]}=o_{p}(1) \\
\delta^{(1-(m-1) r) / 2}|\log \delta|\left[Z_{\delta}, \ldots, Z_{\delta}\right]^{[r, \ldots, r]}\left[\left(\begin{array}{c}
m \\
1
\end{array}\right)\right]=o_{p}(1) \\
\ldots \ldots . \\
\delta^{(1-r) / 2}|\log \delta|^{m-1}\left[Z_{\delta}\right]^{[r]}\left[\left(\begin{array}{c}
m \\
1
\end{array}\right)\right]=o_{p}(1) .
\end{gathered}
$$

These sufficient conditions are also close to being necessary, as the examples below will show.

\subsection{Lévy jumps}

Now, suppose that the jump process $Z$ is a Lévy process. Alternatively, we might consider the case of $Z$ being an OU process with BDLP (background driving Lévy process) L. However, as shown in Barndorff-Nielsen, Shephard, and Winkel (2004), the conclusions regarding CiP and CLT for $X=Y+Z$ would be the same as for $X=Y+L$.

Example 1. Let $Z$ be the $\Gamma(\nu, \alpha)$ subordinator, i.e. $Z$ is the Lévy process for which the law of $Z_{1}$ is the gamma distribution with $p d f$

$$
\frac{\alpha^{\nu}}{\Gamma(\nu)} x^{\nu-1} e^{-\alpha x}
$$

This is an infinite activity process and for $t \downarrow 0$ we have

$$
\mathrm{E}\left\{\left|Z_{t}\right|^{p}\right\}=\alpha^{-p} \frac{\Gamma(t \nu+p)}{\Gamma(t \nu)} \sim O(t)
$$

whatever the value of $p>0$. (Here we have used that $t \Gamma(t) \rightarrow 1$ as $t \rightarrow 0$.) Thus $\left[Z_{\delta}\right]^{[r]}=O_{p}(1)$, $\left[Z_{\delta}, Z_{\delta}\right]^{[r, r]}=O_{p}(\delta),\left[Z_{\delta}, Z_{\delta}, Z_{\delta}\right]^{[r, r, r]}=O_{p}\left(\delta^{2}\right)$, etc.

Consequently:

- MPVCiP is valid for all $m=1,2, \ldots$ and $0<r<2$.

- MPVCLT is valid for all $m=1,2, \ldots$ and $0<r<1$.

On the other hand we have, for example, that BPVCLT does not hold if $r=1$ and $Y \Perp Z$. 
Example 2. Let $Z$ be the $I G(\phi, \gamma)$ subordinator, i.e. $Z$ is the Lévy process for which the law of $Z_{1}$ is the inverse Gaussian distribution with pdf

$$
\frac{\delta}{\sqrt{2 \pi}} e^{\delta \gamma} x^{-3 / 2} e^{-\frac{1}{2}\left(\phi^{2} x^{-1}+\gamma^{2} x\right)}
$$

Then, as $t \downarrow 0$,

$$
\mathrm{E}\left\{\left|Z_{t}\right|^{p}\right\} \sim\left\{\begin{array}{clc}
O(t) & \text { if } & p>\frac{1}{2} \\
O(t|\log t|) & \text { if } & p=\frac{1}{2} \\
O\left(t^{2 p}\right) & \text { if } & 0<p<\frac{1}{2}
\end{array}\right.
$$

so that, for $\frac{1}{2}<r<1$ we have $\left[Z_{\delta}\right]^{[r, r]}=O_{p}(\delta)$ and $\left[Z_{\delta}\right]^{[r]}=O_{p}(1)$. Consequently:

- MPVCiP is valid for all $m=1,2, \ldots$ and $0<r<2$.

- MPVCLT is valid for all $m$ if $\frac{1}{2}<r<1$.

In particular, MPVCLT holds for tripower variation with $r=\frac{2}{3}$.

Example 3. Let $Z$ be the $N I G(\gamma, 0,0, \phi)$ Lévy process. This is representable as the subordination of a Brownian motion $B$ by the $I G(\phi, \gamma)$ subordinator. Hence, $E\left\{\left|Z_{t}\right|^{q}\right\}$ behaves asymptotically as in (6) with $p=q / 2$. Consequently:

- MPVCiP is valid for all $m=1,2, \ldots$ and $0<r<2$.

- MPVCLT does not hold for any value of $r$.

What decides the possibility of MPVCiP or MPVCLT holding is essentially the degree of singularity at 0 of the Lévy measure of $Z$ (which may be expressed in terms of the BlumenthalGetoor index). For the three examples above the degrees are respectively $x^{-1}, x^{-3 / 2}$ and $x^{-2}$. In the latter case there are so many small jumps that the process partly resembles a diffusion, and this is what prevents separate inference on the volatility process $\sigma$.

\section{Microstructure noise}

Zhou (1996) seems to be the first paper that manifestly demonstrates the necessity to take microstructure noise into account when drawing inference on the integrated (squared) volatility of the log price process, based on high frequency data. In Andersen, Bollerslev, Diebold, and Labys (2000) this was emphasised further through the introduction of the volatility signature plot, which made it clear that even for five minute lags the influence of the noise is generally appreciable.

However, the precise nature and influence of the noise is far from well understood and this constitutes a topic of strong current interest.

In a recent paper, Zhang, Mykland, and Aït-Sahalia (2003) address the noise problem and proposes a subsampling procedure for estimating the integrated volatility of the log price process. Hansen and Lunde (2004) have initiated a study of how the realised quadratic variation (RQV) may be bias corrected to alleviate the noise effect. See also the work of Bandi and Russell (2003). The latter line of investigation is continued in joint ongoing work between Barndorff-Nielsen, Hansen, Lunde, and Shephard (2004) and Barndorff-Nielsen, Hansen, Lunde, and Shephard (2005). That work considers a general class of kernel estimators of the RQV of the log price process. It is shown, in particular, that the subsampling procedure for estimation of QRV proposed by Ait-Sahalia, Mykland and Zhang is a special case of that class. However the 
main thrust of the Barndorff-Nielsen, Hansen, Lunde, and Shephard (2004) work consists in determining, from optimality criteria, another type of kernel estimator that has turned out to yield very accurate estimates for almost all lags. The relevance of MPV for the study of microstructure noise will also be considered. In some stimulating recent work Zhang (2004) has shown that subsampling can be generalised to achieve the same rate of convergence as the modified kernel suggested by Barndorff-Nielsen, Hansen, Lunde, and Shephard (2004).

\section{Acknowledgments}

Ole E. Barndorff-Nielsen's work is supported by CAF (www.caf.dk), which is funded by the Danish Social Science Research Council. Neil Shephard's research is supported by the UK's ESRC through the grant "High frequency financial econometrics based upon power variation."

\section{References}

Andersen, T. G., T. Bollerslev, F. X. Diebold, and P. Labys (2000). Great realizations. Risk 13, $105-108$.

Bandi, F. M. and J. R. Russell (2003). Microstructure noise, realized volatility, and optimal sampling. Unpublished paper presented at the Realized volatility conference, Montreal, 8th November, 2003.

Barndorff-Nielsen, O. E., S. E. Graversen, J. Jacod, M. Podolskij, and N. Shephard (2004). A central limit theorem for realised power and bipower variations of continuous semimartingales. Unpublished paper: Nuffield College, Oxford.

Barndorff-Nielsen, O. E., P. R. Hansen, A. Lunde, and N. Shephard (2004). Regular and modified kernel-based estimators of integrated variance: the case with independent noise. Unpublished paper: Nuffield College, Oxford.

Barndorff-Nielsen, O. E., P. R. Hansen, A. Lunde, and N. Shephard (2005). Kernel-based estimators of integrated variance: dependent noise. In preparation.

Barndorff-Nielsen, O. E., J. Jacod, and N. Shephard (2004). Limit theorems for realised bipower variation in econometrics. Unpublished paper: Nuffield College, Oxford.

Barndorff-Nielsen, O. E. and N. Shephard (2001). Non-Gaussian Ornstein-Uhlenbeck-based models and some of their uses in financial economics (with discussion). Journal of the Royal Statistical Society, Series B 63, 167-241.

Barndorff-Nielsen, O. E. and N. Shephard (2002). Econometric analysis of realised volatility and its use in estimating stochastic volatility models. Journal of the Royal Statistical Society, Series B 64, 253-280.

Barndorff-Nielsen, O. E. and N. Shephard (2003). Realised power variation and stochastic volatility. Bernoulli 9, 243-265. Correction published in pages 1109-1111.

Barndorff-Nielsen, O. E. and N. Shephard (2004a). Econometric analysis of realised covariation: high frequency covariance, regression and correlation in financial economics. Econometrica 72, 885-925.

Barndorff-Nielsen, O. E. and N. Shephard (2004b). Power and bipower variation with stochastic volatility and jumps (with discussion). Journal of Financial Econometrics 2, 1-48.

Barndorff-Nielsen, O. E. and N. Shephard (2005). Power variation and time change. Theory of Probability and Its Applications. Forthcoming. 
Barndorff-Nielsen, O. E., N. Shephard, and M. Winkel (2004). Limit theorems for multipower variation in the presence of jumps in financial econometrics. Unpublished paper: Nuffield College, Oxford.

Ghysels, E., A. C. Harvey, and E. Renault (1996). Stochastic volatility. In C. R. Rao and G. S. Maddala (Eds.), Statistical Methods in Finance, pp. 119-191. Amsterdam: North-Holland.

Hansen, P. R. and A. Lunde (2004). An unbiased measure of realized variance. Unpublished paper: Aarhus University.

Mykland, P. and L. Zhang (2005). ANOVA for diffusions. Annals of Statistics 33. Forthcoming.

Shephard, N. (2005). Stochastic Volatility: Selected Readings. Oxford: Oxford University Press. Forthcoming.

Woerner, J. (2004). Power and multipower variation: inference for high frequency data. Unpublished paper.

Zhang, L. (2004). Efficient estimation of stochastic volatility using noisy observations: a multiscale approach. Unpublished paper: Department of Statistics, Carnegie Mellon University.

Zhang, L., P. Mykland, and Y. Aït-Sahalia (2003). A tale of two time scales: determining integrated volatility with noisy high-frequency data. Unpublished paper presented at the Realized volatility conference, Montreal, 8th November 2003.

Zhou, B. (1996). High-frequency data and volatility in foreign-exchange rates. Journal of Business and Economic Statistics 14, 45-52. 